Check for updates

Cite this: RSC Adv., 2019, 9, 26691

Received 23rd June 2019

Accepted 14th August 2019

DOI: $10.1039 / c 9 r a 04723 a$

rsc.li/rsc-advances

\section{The mechanical properties, microstructures and mechanism of carbon nanotube-reinforced oil well cement-based nanocomposites $\uparrow$}

\author{
Shichao Lu, (D) ${ }^{a}$ Xiaoyan Wang, (D) ab Zhaorui Meng, (D) a Qingchun Deng, (D) ${ }^{a}$ \\ Fangfang Peng, (D) a Chengcheng Yu, (D) ${ }^{a}$ Xu Hu, (D) a Yi Zhao, (D) a Yangchuan Ke (iD) *a \\ and Fengzhong Qi $\mathbb{D}$ *b
}

\begin{abstract}
High performance cement-based nanocomposites were successfully fabricated through the use of oil well cement filled with multiwalled carbon nanotubes (MWCNTs) as reinforcements. The dispersibilities of four dispersing agents for the MWCNTs were investigated and compared. The dispersed morphologies and structural characteristics of the MWCNTs were analyzed via TEM, FTIR and Raman spectroscopy studies. The effects of MWCNT addition on the rheological behavior and fluidity of oil well cement slurry were discussed. The mechanical properties of the cement-based nanocomposites with different MWCNT content values and different curing ages were explored and analyzed. Furthermore, the microstructures of the MWCNT reinforced cementitious nanocomposites were characterized via XRD, SEM, EDS, total porosity and pore size distribution studies. The results demonstrated that the 28 day compressive strength and 28 day flexural strength of the 0.05 wt\% MWCNT cementitious nanocomposite increased by $37.50 \%$ and $45.79 \%$, respectively, compared with a pure cement matrix. The elastic moduli of a 0.05 wt\% MWCNT cementitious sample declined by $19.07 \%$ and $35.39 \%$ under uniaxial and triaxial stress, respectively. XRD and pore structure analysis indicated that the MWCNTs could accelerate the hydration process, increase the amount of hydration products and optimize the pore size distribution within the matrix. Additionally, crack bridging, pulling out, network filling and a calcium-silicate-hydrate $(\mathrm{C}-\mathrm{S}-\mathrm{H})$ phase were exhibited by SEM images. Meanwhile, the reinforcing and toughening mechanism of MWCNTs was also discussed; these had a beneficial influence on the mechanical properties.
\end{abstract}

\section{Introduction}

At present, geological conditions are becoming more complicated $^{\mathbf{1 - 3}}$ with the continuous exploration and development of tight oil wells and shale gas wells, ultra-deep wells and gas storage wells. The requirements for cementing quality are getting higher and higher. Correspondingly, higher requirements have also been put forward for cementing technology, cementing materials, cement slurry performance and so on. ${ }^{4}$ Cementing materials are an important aspect of excellent cementing quality, and oil well cement is a main component of cementing materials. However, ordinary cement stone is a brittle material and has inherent defects, such as low tensile strength, poor anti-cracking performance, and low impact

${ }^{a}$ Nanochemistry Key Laboratory of China National Petroleum Corporation, College of Science, China University of Petroleum, Beijing, 102249, China.E-mail: kyc06@sohu. com

${ }^{b}$ CNPC Drilling Research Institute, Beijing, 102206, China. E-mail: qfz69dri@sohu. com

$\dagger$ Electronic supplementary information (ESI) available. See DOI: 10.1039/c9ra04723a strength. ${ }^{5}$ These defects are likely to cause the destruction of cement rings under an impact load, so that their ability to block underground oil, gas and water layers is weakened, resulting in interlayer channeling and the corrosion of casing pipes. ${ }^{6}$ These shortcomings not only severely limit the overall performance and durability of oil well cement, but also lead to wells being scrapped in serious circumstances.

In order to overcome the aforementioned disadvantages of cementing materials, the concept of introducing additional material at the millimeter/micron scale has been advanced in recent years. Pozzolanic additives (metakaolinite, microsilica, etc.) $)^{7,8}$ and fibers (steel fibers, ${ }^{\mathbf{9 - 1 1}}$ glass fibers, ${ }^{\mathbf{1 2 , 1 3}}$ natural fibers $^{\mathbf{1 4 , 1 5}}$ and synthetic fibers ${ }^{\mathbf{1 6}, 17}$ ) at the millimeter/micron scale are commonly-used materials to reinforce cement matrices. Researchers recognize that each kind of additional reinforcement material possesses its own unique enhancement functionality in terms of strength, ductility, crack-control, and so on. Nevertheless, such reinforcement at the millimeter/ micron scale can only limit or delay the expansion of internal micro-cracks in materials, instead of limiting the generation of micro-cracks or restraining nano-cracks. ${ }^{18,19}$ Cementitious 
composites, however, exhibit flaws at the nano-scale, where traditional reinforcement is not effective. ${ }^{20}$

With the development of innovative nanotechnology, dispersing nanomaterials into cement-based composites to develop crack-free concrete with improved mechanical properties and durability is a strategy that has received considerable attention worldwide in recent years. Nanoscale graphene oxide (GO), ${ }^{21}$ nano- $\mathrm{SiO}_{2},{ }^{22}$ nano- $\mathrm{TiO}_{2},{ }^{23}$ nano-MgO ${ }^{24}$ nano- $\mathrm{Al}_{2} \mathrm{O}_{3},{ }^{25}$ nano- $\mathrm{Fe}_{2} \mathrm{O}_{3},{ }^{26}$ nano- $\mathrm{CaCO}_{3},{ }^{27}$ and so on, have opened up new opportunities for the nanosized reinforcement of concrete, ${ }^{28}$ where a small amount of additive can improve the mechanical performance of a cementitious composite. Compared with other nano-materials, carbon nanotubes (CNTs) are considered one of the most ideal nanomaterials for nano-reinforcement, owing to their excellent mechanical, thermal and electrical properties..$^{29,30}$ CNTs are hollow tubular channels, which are commonly categorized as either single-walled carbon nanotubes (SWCNTs) or multi-walled carbon nanotubes (MWCNTs) based on the number of concentric tubes. ${ }^{31}$ MWCNTs can have diameters ranging from $1 \mathrm{~nm}$ to $100 \mathrm{~nm}$, while their lengths range from 0.1 to $100 \mu \mathrm{m} .{ }^{32,33}$ Their aspect ratios (length-todiameter ratios) are beyond 1000 in general. ${ }^{33}$ Meanwhile, the tensile strength of MWCNTs is between $11 \mathrm{GPa}$ and $63 \mathrm{GPa}$, which is 100 times greater than that of steel. ${ }^{34}$ Theoretically, the Young's modulus of MWCNTs approaches $1 \mathrm{TPa}^{35}$ with an ultimate strain of up to $15 \%{ }^{30}$ Different from microfiber reinforcements in cement matrices, which contribute to delays in the development of microcracks, MWCNTs, which are distributed on a much finer scale, prevent crack growth at the nanoscale and limit the initiation of micro-cracks. ${ }^{36}$

In the last few years, many investigation $\mathrm{s}^{34,37-39}$ have focused on adding MWCNTs into cement-based composites for the purpose of strength improvement and microstructure reinforcement. Kumar et $a .^{40}$ explored the effects of MWCNTs on the strength characteristics of hydrated Portland cement paste. Konsta-Gdoutos et $a l^{20}$ found that the incorporation of MWCNTs obviously affected the nano-micron mechanical properties of a cement matrix. Kowald ${ }^{41}$ added $0.50 \mathrm{wt} \%$ MWCNTs into cement paste and measured a $12 \%$ increase in compressive strength. Nochaiya et al. ${ }^{42}$ researched the microstructural properties of MWCNT/cement nanocomposites. SEM micrographs illustrated good interactions between the MWCNTs and cement hydration products, with the MWCNTs acting as a filler and contributing to the formation of a more compact microstructure and higher strength. A major challenge, however, associated with the incorporation of MWCNTs into cement composites is their poor dispersion. Because of the large surface areas and high surface energy of MWCNTs, van der Waals attraction forces are relatively strong between the MWCNTs, meaning that MWCNTs are prone to agglomeration and the formation of bundle structures. ${ }^{19}$ Consequently, the reinforcing effects of MWCNTs on cementitious materials are limited, and their interfacial interaction with the cement matrix is weakened. ${ }^{43}$ Therefore, to achieve favorable reinforcement, the issue of dispersing MWCNTs homogeneously and effectively in the cement matrix is of great importance. ${ }^{44}$ Currently, technologies designed for effectively dispersing MWCNTs are commonly divided into physical dispersion methods and chemical dispersion methods. Physical modification employs mechanical stress through processes such as ultrasonication, ${ }^{45}$ ball milling ${ }^{46}$ and mechanical stirring ${ }^{47}$ to activate the surfaces of MWCNTs. ${ }^{34}$ Chemical modification introduces functional groups to the surfaces of MWCNTs through the use of surfactants, polymers or strong inorganic acid treatment. Simone Musso et $a l .{ }^{48}$ compared samples obtained via mixing the same cement paste with three different kinds of MWCNTs (as-grown, annealed and carboxyl-functionalized MWCNTs). Their results demonstrated that the chemical-physical properties of the MWCNTs affected the mechanical behaviors of the cement composites, but flexural and compressive measurements of the composites showed an $80 \%$ decrease with the functionalized MWCNTs. Li et al. ${ }^{49}$ showed that the addition of MWCNTs modified using a mixture of $\mathrm{H}_{2} \mathrm{SO}_{4} / \mathrm{HNO}_{3}$ into a plain cement matrix moderately enhanced both the compressive and flexural strengths. Cwirzen et al. ${ }^{50}$ combined polyacrylic acid polymers with sonication treatment to disperse MWCNTs in cement composites. The polyacrylic acid polymers were applied to modify the surfaces of the MWCNTs, to promote bonding between the MWCNTs and cement hydration products. Sáez de Ibarra et $a l .{ }^{43}$ employed arabic gum (a water-soluble surfactant) as a dispersing agent and found improvements in the Young's modulus and compressive strength. Meanwhile, the incorporation of MWCNTs worsened the mechanical properties when no surfactant was used.

Here, in this paper, the effective dispersion of MWCNTs in water was achieved through applying surfactants and, in combination with an ultrasonication and centrifugation process, the dispersions were used to facilitate the preparation of MWCNT-reinforced oil well cement-based nanocomposites. The dispersion performances of the MWCNTs were investigated and four dispersing agents were compared. The dispersion morphologies and structural characteristics of the MWCNTs were analyzed via TEM, FTIR and Raman spectroscopy studies. Moreover, the effects of the MWCNT content on the rheological behavior and fluidity of the oil well cement slurry were discussed. In addition, the compressive strength and flexural strength of oil well cement-based nanocomposites with different MWCNT content values and different curing ages were studied, and uniaxial and triaxial mechanical properties were measured to evaluate the effects of the MWCNTs on the elastic modulus values. Meanwhile, the microstructures of the MWCNT reinforced cementitious nanocomposites were characterized via XRD, SEM, EDS, total porosity and pore size distribution studies. Finally, the strengthening and toughening mechanism of the cement composites with MWCNTs was also analyzed. The results from this paper can lead to further understanding of MWCNT/cement nanocomposites in the petroleum engineering field.

\section{Experimental}

\subsection{Materials}

The class $G$ oil well cement used for this experiment was manufactured by Sichuan Jiahua Enterprise (group) Co., Ltd 
(Sichuan, China). The MWCNTs were supplied by Beijing DK Nano Technology Co., Ltd (Beijing, China). MWCNT dispersant (TNWDIS) was obtained from Chengdu Organic Chemicals Co., Ltd. (Chinese Academy of Sciences). TNWDIS is an aromatic modified polyethylene glycol ether. ${ }^{36}$ The aromatic rings of TNWDIS are hydrophobic and connected to hydrophilic groups through carbon chains. The other MWCNT dispersing agents, sodium dodecylbenzene sulfonate (SDBS), arabic gum (AG or $\mathrm{GA}$ ), and polyvinyl pyrrolidone (PVP), were purchased from Aladdin Reagents (Shanghai) Co., Ltd. The antifoaming agent DRX-1L was provided by the China National Petroleum Corporation Drilling Research Institute. The experimental water used was tap water.

\subsection{Preparation}

2.2.1 Preparation of MWCNT suspensions. For the preparation of MWCNT suspensions, antifoaming agent (20-40 wt\% of the dispersing agent weight) was first added to water, followed by the addition of the dispersing agent (surfactant) and even magnetic stirring. Then, a designated amount of MWCNT powder was placed in this aqueous solution and stirred at a constant speed of $200 \mathrm{rpm}$ for $20 \mathrm{~min}$. The content of surfactant added was approximately $20 \%$ of the weight of the MWCNTs. After that, the resulting suspension was treated under ultrasonication for $30 \mathrm{~min}$. To prevent overheating and foaming, the suspension was placed in an ice-water bath during the sonication process. Subsequently, the MWCNT suspension was centrifuged for $30 \mathrm{~min}$ at a centrifugal speed of $5000 \mathrm{rpm}$, to collect agglomerates of MWCNTs. After centrifugation, the MWCNT agglomerates were re-diluted, sonicated, and centrifuged until the bundles of MWCNTs were separated into individual strands. ${ }^{34}$

2.2.2 Manufacture of MWCNT cementitious nanocomposites. A water-to-cement (w/c) ratio of 0.44 was utilized in all specimens. The amounts of MWCNTs in the cementitious nanocomposites were $0.00 \mathrm{wt} \%, 0.025 \mathrm{wt} \%, 0.05 \mathrm{wt} \%, 0.10 \mathrm{wt} \%$, $0.25 \mathrm{wt} \%, 0.50 \mathrm{wt} \%, 1.0 \mathrm{wt} \%$ and $2.0 \mathrm{wt} \%$ by weight of cement. Class $\mathrm{G}$ oil well cement was added into the prepared MWCNT suspensions. The blend was mixed using a standard Hobart mixer, according to the ASTM C305 standard. ${ }^{51}$ The mixture was stirred at a high speed for $300 \mathrm{~s}$. After mixing, the MWCNT cementitious samples were cast into molds $(40 \mathrm{~mm} \times 40 \mathrm{~mm} \times$ $160 \mathrm{~mm}$ or $50 \mathrm{~mm} \times 50 \mathrm{~mm} \times 50 \mathrm{~mm}$ ) and an electric vibrator was used to ensure good compaction. The samples were demolded after $24 \mathrm{~h}$ and cured in water under standard curing conditions until the testing age was reached.

\subsection{Characterization}

2.3.1 Rheological behavior. A six-speed rotational viscometer (Qingdao Haitongda Special Instrument Co., Ltd) was used to calculate the rheological parameters of MWCNT/oil well cement slurries. The formulas for calculating the flow index (1) and consistency coefficient (2) were as follows:

$$
n=2.096 \times\left[\lg \left(\theta_{300} / \theta_{100}\right)\right]
$$

$$
K=0.511 \times\left(\theta_{300} / 511^{n}\right)
$$

where $\theta_{300}$ and $\theta_{100}$ are the values when the speeds are $300 \mathrm{rpm}$ and $100 \mathrm{rpm}, n$ is the flow index and $K(\mathrm{~Pa} \mathrm{~s})$ is the consistency coefficient.

2.3.2 Fluidity evaluation. A truncated cone round mold was used to measure the fluidity of MWCNT/oil well cement slurries. In the experiment, the truncated cone round mold was placed on a horizontal glass plate, newly prepared cement slurry was quickly poured into the truncated cone round mold, and then the truncated cone round mold was raised vertically. Finally, the maximum horizontal spread diameter of the cement slurry was measured, and the average value from three specimens was defined as the fluidity $d(\mathrm{~cm})$.

2.3.3 Compressive strength measurements. Compressive strength tests were performed according to ASTM C349. ${ }^{52}$ The compressive strength value was measured using a compressive strength tester (Shenyang Gold OUKE Petroleum Instrument Technology Development Co., Ltd). Three hardened MWCNT/ cement samples were tested and the results were averaged.

2.3.4 Flexural strength measurements. The flexural strength tests conducted in this paper were mainly performed according to ASTM C348. ${ }^{53}$ An RGM-X300 universal pressure tester (Shenzhen Reger Instrument Co., Ltd) was used in the flexural strength tests. Three specimens were subjected to measurements and average values were reported. The formula (3) for calculating the flexural strength was as follows:

$$
R_{\mathrm{f}}=(3 P L) /\left(2 b h^{2}\right)
$$

where $R_{\mathrm{f}}(\mathrm{MPa})$ is the flexural strength, $P(\mathrm{~N})$ is the maximum force value at the instant of failure in a bent beam, $L$ is the span value between two supports $(100 \mathrm{~mm})$, and $b$ and $h$ are the width and height, respectively (both $40 \mathrm{~mm}$ ).

2.3.5 Uniaxial and triaxial mechanical experiments. A TAW-1000 microcomputer controlled electro-hydraulic servo cement triaxial pressure testing machine (Changchun Chaoyang Test Instrument Co., Ltd) was used for testing. The cement sample was a cylinder with a diameter of $25 \mathrm{~mm}$ and a height of $50 \mathrm{~mm}$. Both ends of the cylindrical specimen were flat and polished, the base surface deviation was within $2.5 \%$, and the length-diameter ratio of the specimen was $\geq 1.5$.

2.3.6 Microstructural analysis. Fourier transform infrared spectroscopy (FTIR) studies of the MWCNT suspensions were performed using an FTS-3000 spectrophotometer (American Digilab Company) from 4000 to $500 \mathrm{~cm}^{-1}$.

Raman spectroscopy data were obtained by means of a Renishaw inVia Plus Raman system (Renishaw Company, London, UK), and an excitation wavelength of $514.5 \mathrm{~nm}$ was generated using an Argon laser.

The phase composition analysis of the MWCNT-reinforced cementitious nanocomposites was carried out using Bruker D8 Advance X-ray diffraction (XRD) apparatus (Bruker Company, Karlsruhe, Germany) with $\mathrm{Cu}-\mathrm{K} \alpha$ radiation $(\lambda=$ $1.540598 \AA$ ) over the scattering range $(2 \theta)$ of $5-70^{\circ}$ with a scanning speed of $4^{\circ} \mathrm{min}^{-1}$ and a scanning interval of $0.02^{\circ}$. The powder samples for XRD measurements were obtained via 
crushing fragments of broken specimens after the compression tests.

The morphology of the fracture surface was characterized via scanning electron microscopy (SEM, S-4800, Hitachi, Japan). Fractured specimens were soaked in absolute ethanol to prevent hydration. Before SEM testing, the fragments were removed from ethanol and dried in an oven to a constant weight. Also, energy dispersive spectrometry (EDS) was employed to determine the elemental compositions of the cement composites.

The dispersion morphologies of the MWCNTs were observed using transmission electron microscopy (TEM, JEM-2100, Tokyo, Japan) at an accelerating voltage of $200 \mathrm{kV}$.

The total porosity and pore size distribution data from cement samples were analyzed via mercury intrusion porosimetry (MIP, AutoPore IV 9500). Samples for tests were cured under standard conditions for 28 days and broken into 3-6 $\mathrm{mm}$ particles. Then, the samples were dried at $100{ }^{\circ} \mathrm{C}$ for $12 \mathrm{~h}$ before MIP measurements.

\section{Results and discussion}

\subsection{Dispersibility of MWCNT suspensions}

3.1.1 The optimization of the MWCNT dispersing agent. The dispersants used in the experiments were SDBS, TNWDIS, GA and PVP. As shown in Table 1, MWCNTs were added into different kinds of dispersant solutions. The standing time of the MWCNT suspensions was $0 \mathrm{~h}, 4 \mathrm{~h}, 16 \mathrm{~h}$ and $32 \mathrm{~h}$ after ultrasonic dispersion treatment; evaluation of the dispersants was carried out by observing the degree of dark color observable in the suspensions and the precipitates (Fig. S1, see ESI $\uparrow$ for details). At the same time, in order to further investigate the stabilities of the MWCNT suspensions, the MWCNT suspensions were centrifuged at a centrifugal speed of $4000 \mathrm{rpm}$. The centrifugation time required for stratification was recorded.

Fig. 1 shows the centrifugation time required to stratify MWCNTs in different dispersants. The results demonstrate that it takes the longest time to stratify a suspension of MWCNTs dispersed in TNWDIS, and delamination occurred after 200 minutes, showing that the suspension has excellent stability. GA is second, PVB and SDBS show successively decreased times, and water is the worst. Therefore, the addition of a dispersant facilitates the dispersion of the MWCNTs in the aqueous system, and different dispersing agents have different dispersion effects. TNWDIS can be absorbed on the surfaces of

Table 1 MWCNT dispersions in different dispersants

\begin{tabular}{|c|c|c|c|c|c|c|}
\hline Number & Water (g) & $\begin{array}{l}\text { MWCNTs } \\
(\mathrm{g})\end{array}$ & SDBS $(g)$ & PVP (g) & GA (g) & $\begin{array}{l}\text { TNWDIS } \\
(\mathrm{g})\end{array}$ \\
\hline 1 & 20 & 0.04 & 0 & 0 & 0 & 0 \\
\hline 2 & 20 & 0.04 & 0.08 & 0 & 0 & 0 \\
\hline 3 & 20 & 0.04 & 0 & 0.08 & 0 & 0 \\
\hline 4 & 20 & 0.04 & 0 & 0 & 0.08 & 0 \\
\hline 5 & 20 & 0.04 & 0 & 0 & 0 & 0.08 \\
\hline
\end{tabular}

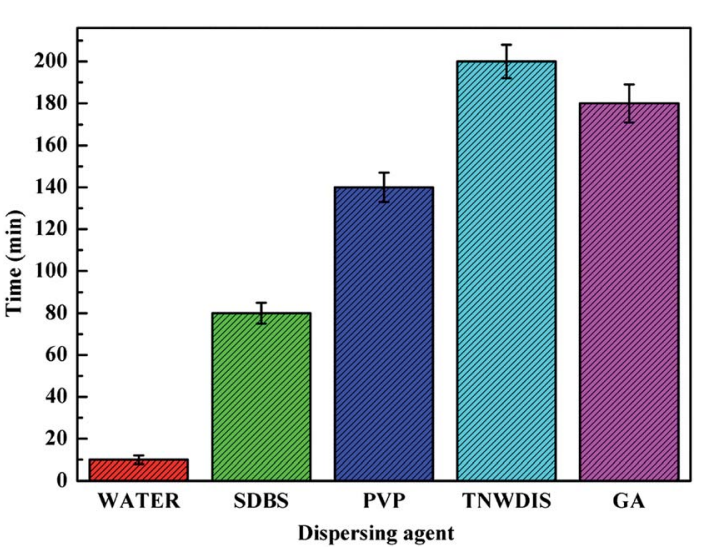

Fig. 1 Centrifugal time for different MWCNT suspensions.

MWCNTs, forming micelles around the MWCNTs and inhibiting their aggregation. The micelles formed during this process contribute to exfoliating individual MWCNTs from bundles through electrostatic repulsion or steric hindrance effects. ${ }^{30}$ The results indicate that TNWDIS should be selected as the dispersant for MWCNTs due to it showing the best dispersion effects.

3.1.2 Characterization of MWCNTs dispersed with TNWDIS. The dispersion morphology and structural characteristics of MWCNTs dispersed in TNWDIS are analyzed via TEM, FTIR spectra and Raman spectroscopy. Fig. 2a-b shows TEM images of the MWCNT suspension. MWCNT agglomerates can be observed before the addition of TNWDIS, as shown in Fig. 2a. After dispersion with TNWDIS, the MWCNTs are dispersed effectively and homogeneously in the suspension (Fig. 2b). The results indicate that the addition of TNWDIS can facilitate the dispersion of MWCNTs in an aqueous suspension. Because the surface treatment of MWCNTs with TNWDIS changes the surface polarity of the MWCNTs and weakens van der Waals forces, this leads to efficient debundling and prohibits MWCNTs from re-aggregating. Fig. 3 presents the FTIR spectrum of the MWCNT suspension. An absorption peak appearing at $1630 \mathrm{~cm}^{-1}$ is related to the $\mathrm{C}=\mathrm{C}$ stretching vibration of the graphite structure in the MWCNT framework. ${ }^{54}$ This is caused by the five-membered ring or sevenmembered ring at the turning point or seal of the MWCNTs. The peak at $3420 \mathrm{~cm}^{-1}$ is assigned to the $-\mathrm{OH}$ stretching vibration of TNWDIS. The peaks at $2930 \mathrm{~cm}^{-1}$ and $2850 \mathrm{~cm}^{-1}$ are ascribed to the stretching vibrations of $-\mathrm{CH}_{2}$ - and $-\mathrm{CH}_{3}$ groups in TNWDIS. The peaks at $1500 \mathrm{~cm}^{-1}$ and $1450 \mathrm{~cm}^{-1}$ correspond to the $\mathrm{C}=\mathrm{C}$ stretching of the aromatic structure deriving from TNWDIS, and the observed peaks at $754 \mathrm{~cm}^{-1}$ and $690 \mathrm{~cm}^{-1}$ are characteristic of the benzene ring. Peaks from $\mathrm{C}-\mathrm{O}-\mathrm{C}$ stretching are observed at $1384 \mathrm{~cm}^{-1}$ and $1109 \mathrm{~cm}^{-1}$ in TNWDIS. The Raman spectrum of MWCNTs (Fig. S2 $\dagger$ ) demonstrates two characteristic peaks. One peak at $1340 \mathrm{~cm}^{-1}$ is attributed to the disorder-induced mode (D band). The other peak at $1610 \mathrm{~cm}^{-1}$ is ascribed to the graphite mode ( $G$ band) in the MWCNT structure. ${ }^{48}$ In addition, the ID/ IG ratio is 1.69 , suggesting that the incorporation of TNWDIS 

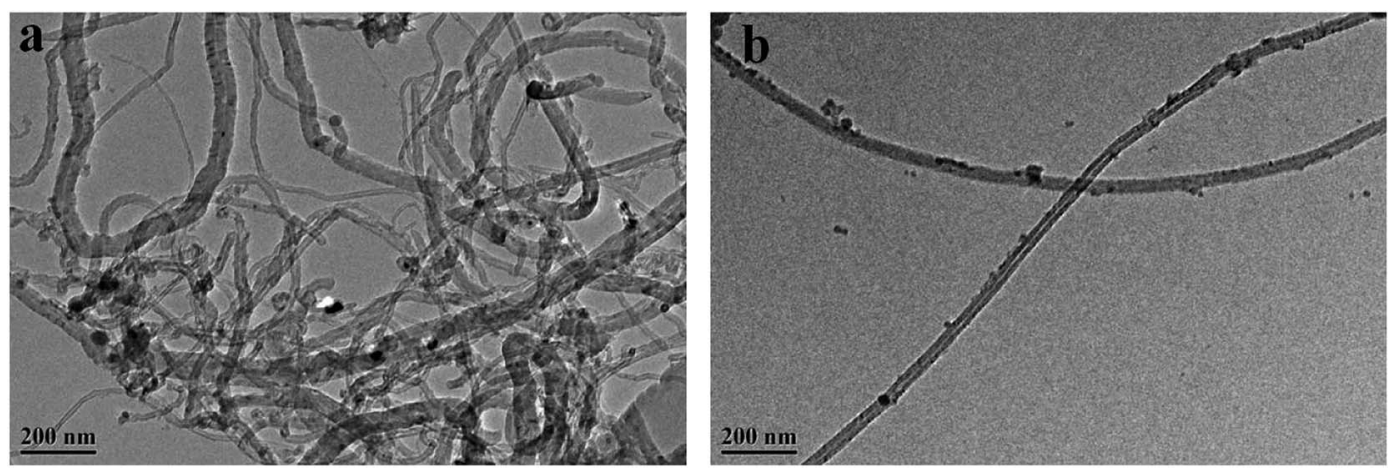

Fig. 2 TEM images of MWCNTs dispersed with TNWDIS: (a) before dispersion; and (b) after dispersion.

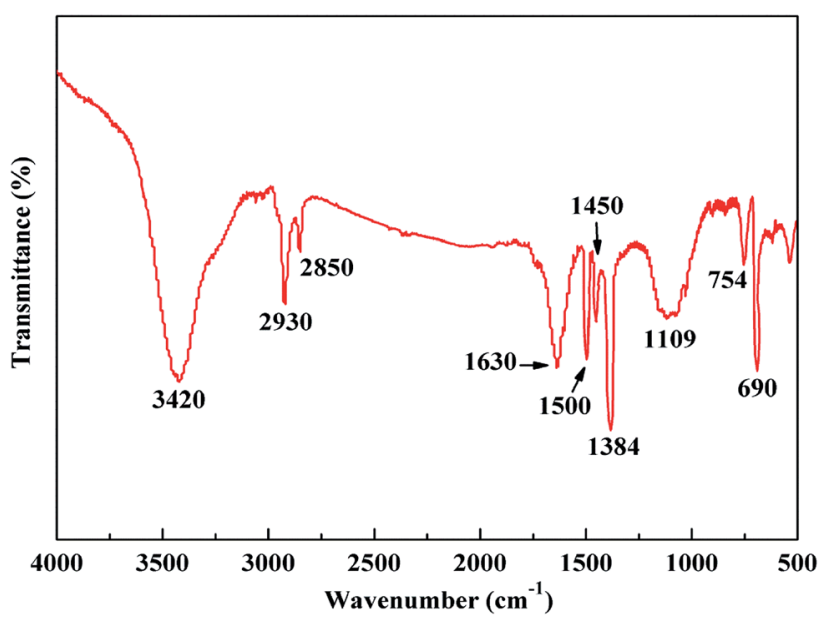

Fig. 3 The FTIR spectrum of a MWCNT suspension.

increases lattice disorder in the MWCNTs and modifies the MWCNT surfaces.

\subsection{The effects of MWCNTs on the rheological properties of oil well cement slurry}

In order to investigate the effects of the addition of different amounts of MWCNTs on the rheological properties of oil well cement slurry, rheological measurements were performed using a six-speed rotational viscometer. The experimental results are shown in Table 2. The water-to-cement ratio was 0.44. MWCNTs were dispersed with TNWDIS. The TNWDIS content added was approximately $20 \%$ of the weight of the MWCNTs. The shear stress values from left to right correspond to readings at rotational speeds of $600 \mathrm{rpm}, 300 \mathrm{rpm}, 200 \mathrm{rpm}, 100 \mathrm{rpm}, 6 \mathrm{rpm}$ and $3 \mathrm{rpm}$, respectively.

The results in Table 2 indicate that all slurries exhibit obvious shear thinning. The flow index $n$ decreases slowly, and the consistency coefficient $K$ increases with an increase in the MWCNT content. The addition of MWCNTs has a great influence on the rheological properties of oil well cement slurry. When the MWCNT content added is controlled between $0.05 \mathrm{wt} \%$ and $1.0 \mathrm{wt} \%$, the rheological properties of the cement slurry are favorable and satisfy construction requirements, indicating that an optimum mass concentration range of MWCNTs is needed to achieve the ideal rheological effects. However, when the addition of MWCNTs exceeds $1.0 \mathrm{wt} \%$, the dispersion degree of the MWCNTs declines and more MWCNTs tend to agglomerate, so the rheological properties are affected obviously.

\subsection{The effects of MWCNTs on the fluidity of oil well cement slurry}

In an oil well cementing project, the fluidity of the cement slurry is generally required to be higher than $18 \mathrm{~cm}$ in order to facilitate pumping. The make-up of the oil well cement slurry used in these experiments is identical to that used in the above section. The fluidity experimental results are shown in Fig. 4. The incorporation of MWCNTs into the cement slurry reduces the fluidity of the cement slurry. The fluidity declines gradually with an increase in the amount of MWCNTs. The reason for this is that MWCNTs act as nucleation sites to accelerate

Table 2 The effects of MWCNTs on the rheological properties of oil well cement slurry

\begin{tabular}{llllll}
\hline Number & $\begin{array}{l}\text { MWCNT content } \\
(\mathrm{wt} \%)\end{array}$ & $\begin{array}{l}\text { Cement slurry } \\
\text { density }\left(\mathrm{g} \mathrm{cm}^{-3}\right)\end{array}$ & $\begin{array}{l}\text { Cement slurry } \\
\text { volume }\left(\mathrm{cm}^{3}\right)\end{array}$ & Shear stress values & $K($ Pa s) \\
\hline 1 & 0.00 & 1.90 & 350 & $96 / 75 / 61 / 47 / 25 / 20$ & 0.43 \\
2 & 0.05 & 1.90 & 350 & $65 / 51 / 36 / 20 / 8 / 2$ & 2.70 \\
3 & 0.25 & 1.90 & 350 & $70 / 36 / 26 / 16 / 5 / 3$ & 0.85 \\
4 & 0.50 & 1.90 & 350 & $104 / 65 / 54 / 31 / 20 / 10$ & 0.74 \\
5 & 1.0 & 1.90 & 350 & $140 / 100 / 72 / 49 / 17 / 13$ & 0.13 \\
6 & 2.0 & 1.90 & 350 & $160 / 121 / 103 / 64 / 19 / 13$ & 0.65 \\
\end{tabular}




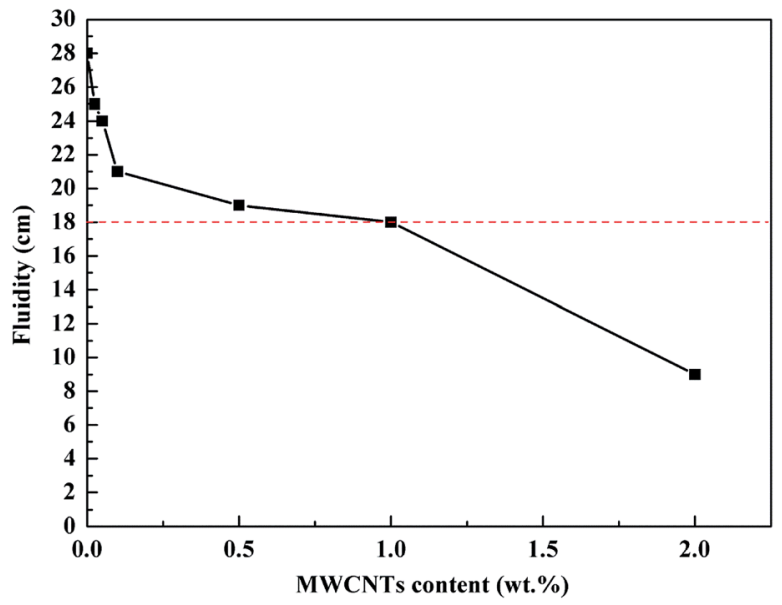

Fig. 4 The effects of MWCNTs on the fluidity of oil well cement slurry.

the hydration reaction. ${ }^{55}$ When the amount of MWCNTs added is $0.025 \mathrm{wt} \%$, the fluidity is $25 \mathrm{~cm}$, which is beneficial for the fluidity of cement slurry. The fluidity of the cement slurry is kept above $18 \mathrm{~cm}$ as the amount of MWCNTs is controlled at less than $1.0 \mathrm{wt} \%$. A MWCNT content of less than $0.50 \mathrm{wt} \%$ is more economical and suitable, and it can meet the on-site pumping requirements. When the MWCNT dosage is more than $1.0 \mathrm{wt} \%$, the fluidity of the cement slurry is less than $18 \mathrm{~cm}$ and does not meet cementing requirements. Therefore, in order to ensure the smooth pumping of oil well cement slurry, the addition of MWCNTs should be preferably less than $0.50 \mathrm{wt} \%$.

\subsection{Compressive strength of the MWCNT cementitious nanocomposites}

3.4.1 The effects of MWCNTs on the early compressive strength of cementitious nanocomposites. Early compressive strength testing of cementitious nanocomposites with different amounts of MWCNTs were carried out after curing for 1 day and 3 days. The results are shown in Table 3. As can be seen from Table 3, when the amount of MWCNTs added is $0.05 \mathrm{wt} \%$, the compressive strength increases by $18.40 \%$ and $14.39 \%$ after 1 day and 3 days, respectively, compared with the pure sample $\mathrm{P}$. The compressive strength of the cement nanocomposite $\mathrm{C} 2$ after curing for 3 days reaches the maximum value of $30.2 \mathrm{MPa}$. As for the compressive strength of the $0.10 \mathrm{wt} \%$ MWCNT cement specimen C3 after curing for
1 day, it increases by $29.60 \%$ to the maximum value of 16.2 MPa. The compressive strength has a tendency to decrease when the addition of MWCNTs is more than $0.10 \mathrm{wt} \%$. This is because the MWCNTs can't be dispersed well, and they become entangled and entwined with each other during the mixing process. ${ }^{56}$ The results demonstrate that MWCNTs can improve the early compressive strength of cementitious nanocomposites. At the same time, adding a small amount of MWCNTs can better exert the reinforcing effects of MWCNTs on cement.

3.4.2 The effects of curing time on the compressive strength of the MWCNT cementitious nanocomposites. Fig. 5 exhibits the compressive strength changes over the curing period. The compressive strength of a pure cement sample $\mathrm{P}$ appears to increase first and then decreases with an increase in the curing age, and the compressive strength reaches its maximum value after 14 days. Over the curing period of 14 days, the compressive strengths of sample C1 and sample C2 are higher than that of pure cement $\mathrm{P}$, and the compressive strengths of sample C3 and sample C4 are lower than that of pure cement $\mathrm{P}$. The reason for this is that cement composites with a small amount of MWCNTs react faster during the initial stage of hydration, and the hydration rate gradually slows down as the amount of MWCNT addition increases. The 28 day compressive strengths of $\mathrm{C} 1$ and $\mathrm{C} 2$ can be increased by $21.88 \%$ and $37.50 \%$, respectively, compared with pure cement P. The results show that MWCNTs can enhance the

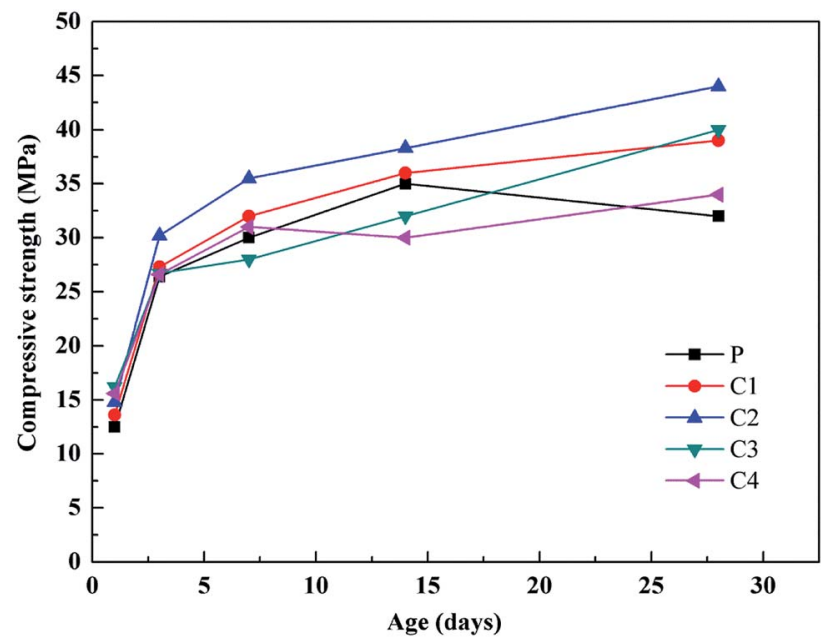

Fig. 5 The effects of curing time on the compressive strength.

Table 3 Early compressive strengths of cementitious nanocomposites

\begin{tabular}{|c|c|c|c|c|c|}
\hline Sample & $\begin{array}{l}\text { Oil well cement } \\
\text { (g) }\end{array}$ & $\begin{array}{l}\text { MWCNT content } \\
(\mathrm{wt} \%)\end{array}$ & $\begin{array}{l}\text { Water-cement } \\
\text { ratio }(\mathrm{w} / \mathrm{c})\end{array}$ & $\begin{array}{l}\text { Compressive strength } \\
\text { (1 day, } \mathrm{MPa})\end{array}$ & $\begin{array}{l}\text { Compressive strength } \\
\text { ( } 3 \text { days, } \mathrm{MPa} \text { ) }\end{array}$ \\
\hline $\mathrm{P}$ & 800 & 0.00 & 0.44 & 12.5 & 26.4 \\
\hline C1 & 800 & 0.025 & 0.44 & 13.6 & 27.3 \\
\hline $\mathrm{C} 2$ & 800 & 0.05 & 0.44 & 14.8 & 30.2 \\
\hline C3 & 800 & 0.10 & 0.44 & 16.2 & 26.7 \\
\hline $\mathrm{C} 4$ & 800 & 0.50 & 0.44 & 15.6 & 26.6 \\
\hline
\end{tabular}


compressive strengths of cement nanocomposites, and their enhancement effects become more obvious with an increase in the curing age.

\subsection{Flexural strength of the MWCNT cementitious nanocomposites}

3.5.1 The effects of MWCNTs on the early flexural strengths of cementitious nanocomposites. Under standard curing conditions, early flexural strength measurements were performed at ages of 1 day and 3 days. The experimental results are displayed in Table 4. The flexural strengths of the MWCNT/ cement samples show a tendency to increase first and then decrease with an increase in the MWCNT content. For the cement sample with $0.10 \mathrm{wt} \%$ MWCNTs, the increases in flexural strength are $22.86 \%$ and $37.25 \%$ at ages of 1 day and 3 days, respectively, compared with the pure cement sample $\mathrm{P}$, and the flexural strengths reach maximums of $4.3 \mathrm{MPa}$ and $7.0 \mathrm{MPa}$, respectively. The flexural strength of sample $\mathrm{C} 4$, containing $0.50 \mathrm{wt} \%$ MWCNTs, is lower than that of the $0.10 \mathrm{wt} \%$ MWCNT sample C3. The agglomeration of MWCNTs at high dosages brings about poor connectivity between the MWCNTs and the cement matrix, which does not result in any increase in flexural strength. The results indicate that a proper amount of MWCNTs can improve the early flexural strengths of cementitious nanocomposites.

3.5.2 The effects of curing time on the flexural strengths of the MWCNTs cementitious nanocomposites. The cementitious nanocomposite samples were cured under standard curing conditions, and the flexural strengths after different periods were tested. From Fig. 6, it can be seen that the flexural strength increases along with an increase in the curing age. In the initial stage of hydration, the hydration rates of the samples containing large amounts of MWCNTs are slow. When the curing time exceeded 7 days, the flexural strength obviously increased. Of the samples, the flexural strengths of sample $\mathrm{C} 1$ and sample $\mathrm{C} 2$ after 14 days are increased by $39.76 \%$ and $49.40 \%$, respectively, compared with that of pure cement $\mathrm{P}$. The flexural strengths of sample $\mathrm{C} 2$ and sample C3 after 28 days are higher than that of the pure sample $\mathrm{P}$ by $45.79 \%$ and $42.99 \%$, respectively. The experimental results show that the flexural strengths of MWCNT/ cement samples are significantly higher than that of pure cement; MWCNTs can play an enhanced role, especially during the later period of hydration. As the hydration reaction proceeds, the excellent mechanical properties of the

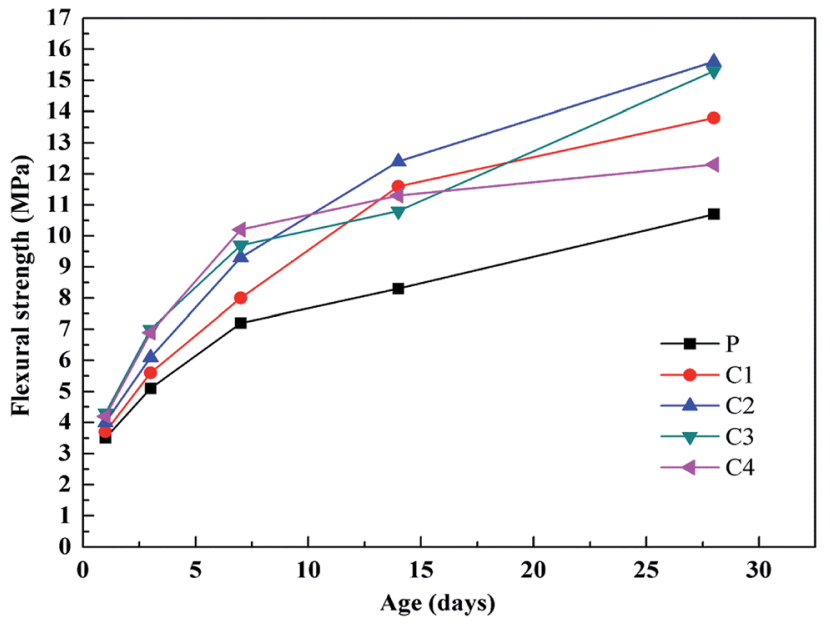

Fig. 6 The effects of curing time on the flexural strength.

MWCNTs themselves are gradually exhibited and, at the same time, they can be well cemented in the cement matrix. Therefore, the flexural strength enhances with an increase in MWCNT addition.

\subsection{Uniaxial and triaxial mechanical properties}

In this experiment, uniaxial and triaxial mechanical property measurements were conducted using the sample C2 (the addition of $0.05 \mathrm{wt} \%$ MWCNTs). A $\Phi 25 \times 50 \mathrm{~mm}$ sample designed for testing was cured in a water bath at $80{ }^{\circ} \mathrm{C}$ for 2 days. The test temperature was $25{ }^{\circ} \mathrm{C}$. Sample $\mathrm{P}$ was a pure cement sample, used as a comparative sample. The results are shown in Table 5 and Fig. S3.†

As can be seen in Table 5, the elastic moduli of the cementitious samples decline upon the addition of MWCNTs; meanwhile, the peak strengths decrease within a certain range. Fig. S3a $\uparrow$ shows that the axial strain of the MWCNT/cement sample $\mathrm{C} 2$ is greater than that of the pure sample $\mathrm{P}$ (Fig. S3b $\dagger$ ) when subjected to uniaxial stress. The cementitious nanocomposite is in an elastic deformation stage due to the low stress, and the stress-strain curve is almost linear. It can be calculated that the elastic modulus of the MWCNT/cement sample C2 is $7.17 \mathrm{GPa}$, which is a reduction of $19.07 \%$ compared with the pure sample $\mathrm{P}$. In order to simulate the stress environment downhole, a triaxial stress loading method is adopted. From the triaxial stress-strain curve in Fig. S3c, $\uparrow$ the axial strain of sample $\mathrm{C} 2$ is much higher than that of pure

Table 4 Early flexural strengths of cementitious nanocomposites

\begin{tabular}{|c|c|c|c|c|c|}
\hline Sample & $\begin{array}{l}\text { Oil well cement } \\
(\mathrm{g})\end{array}$ & $\begin{array}{l}\text { MWCNT content } \\
\text { (wt\%) }\end{array}$ & $\begin{array}{l}\text { Water-cement } \\
\text { ratio }(\mathrm{w} / \mathrm{c})\end{array}$ & $\begin{array}{l}\text { Flexural strength } \\
\text { (1 day, } \mathrm{MPa})\end{array}$ & $\begin{array}{l}\text { Flexural strength } \\
\text { ( } 3 \text { days, } \mathrm{MPa})\end{array}$ \\
\hline $\mathrm{P}$ & 800 & 0.00 & 0.44 & 3.5 & 5.1 \\
\hline C1 & 800 & 0.025 & 0.44 & 3.7 & 5.6 \\
\hline $\mathrm{C} 2$ & 800 & 0.05 & 0.44 & 4.0 & 6.1 \\
\hline C3 & 800 & 0.10 & 0.44 & 4.3 & 7.0 \\
\hline $\mathrm{C} 4$ & 800 & 0.50 & 0.44 & 4.2 & 6.9 \\
\hline
\end{tabular}


Table 5 Uniaxial and triaxial mechanical property results from cementitious samples

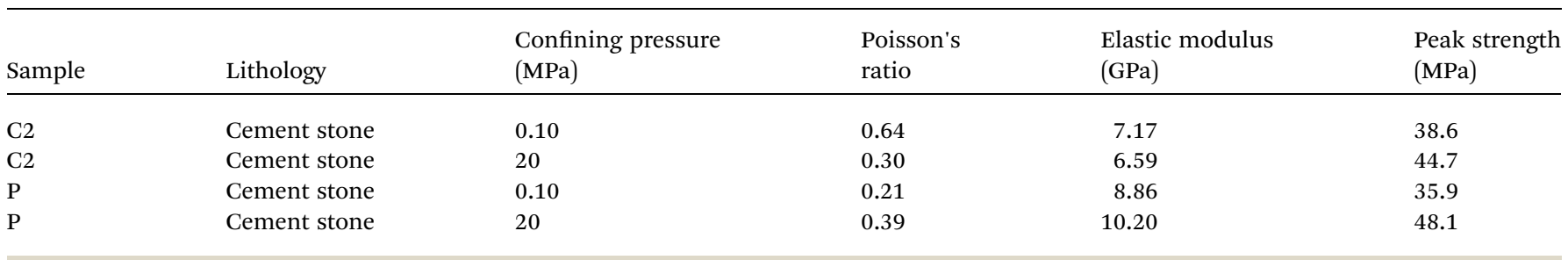

sample P (Fig. S3d $\dagger$ ) under triaxial stress. It can be seen that the elastic modulus $(6.59 \mathrm{GPa})$ is reduced by $35.39 \%$ compared with pure cement $\mathrm{P}$. Sample $\mathrm{P}$ shows typical brittle failure, while sample C2 presents strong plastic deformability. The experimental results indicate that the MWCNTs can effectively reduce the elastic modulus of the cement composite and have a toughening effect on the cement composite.

\subsection{XRD analysis}

The XRD patterns of cement composites with different MWCNT content values, after 28 days of curing, are shown in Fig. 7. Since the added amounts of MWCNTs in the cement matrix are all rather small, there are no characteristic peaks corresponding to MWCNTs in the XRD patterns. Therefore, this figure depicts that none of the samples demonstrate any major differences between their diffraction patterns, indicating that no new phases are formed in the MWCNT cementitious nanocomposites compared with the pure cement sample. However, the intensities of the peaks relating to calcium-silicate-hydrate (C-S-H gel), calcium hydroxide $\left(\mathrm{Ca}(\mathrm{OH})_{2}\right)$, tricalcium silicate $\left(\mathrm{C}_{3} \mathrm{~S}\right)$ and dicalcium silicate $\left(\mathrm{C}_{2} \mathrm{~S}\right)$ change a lot. ${ }^{57}$ The diffraction peaks of $\mathrm{Ca}(\mathrm{OH})_{2}$ are located at $2 \theta$ values of around $18.7^{\circ}, 35^{\circ}$ and $48.5^{\circ}$, respectively. The main peak intensities of $\mathrm{Ca}(\mathrm{OH})_{2}$ at $18.7^{\circ}$ in samples $\mathrm{C} 1, \mathrm{C} 2, \mathrm{C} 3$, and C4 increase by $41.9 \%, 72.5 \%$, $54.4 \%$, and $23.2 \%$, respectively, compared with pure cement $\mathrm{P}$, which illustrates that the incorporation of MWCNTs promotes the cement hydration process. Meanwhile, the $\mathrm{C}-\mathrm{S}-\mathrm{H}$ gel peak

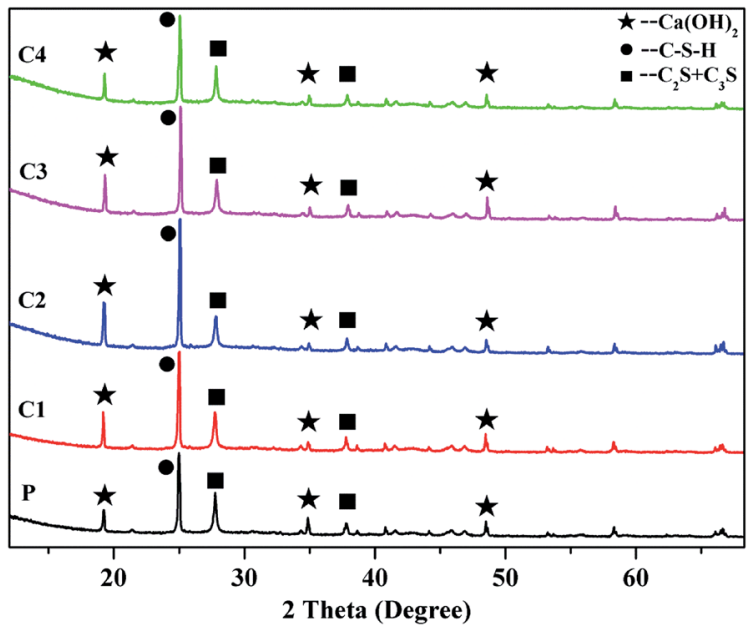

Fig. 7 XRD patterns of different MWCNT/cement samples after 28 days of curing. intensities of samples C1, C2, C3, and $\mathrm{C} 4$ at a $2 \theta$ angle of $25^{\circ}$ are $18.4 \%, 56.1 \%, 32.8 \%$, and $9.6 \%$ higher, respectively, than that of pure cement $\mathrm{P}$. Indeed, such results show a similar tendency to the compressive and flexural strength changes after 28 days. This shows that MWCNTs serve as nucleation sites, so that more $\mathrm{C}-\mathrm{S}-\mathrm{H}$ gel is generated in the hydration product. The dense $\mathrm{C}-\mathrm{S}-\mathrm{H}$ structure can improve the load-transfer capacity between the cement matrix and MWCNTs, thus contributing to the strengths of samples. In addition, the diffraction peaks at $27.5^{\circ}$ and $37.7^{\circ}$ arise from unhydrated products, such as $\mathrm{C}_{3} \mathrm{~S}$ and $\mathrm{C}_{2} \mathrm{~S}$. It can be seen that the peak intensities of $\mathrm{C}_{3} \mathrm{~S}$ and $\mathrm{C}_{2} \mathrm{~S}$ in the MWCNT nanocomposites are lower than in pure cement $\mathrm{P}$ at a $2 \theta$ angle of $27.5^{\circ}$, indicating the higher hydration degree of the MWCNT/cement nanocomposites. The reason for this phenomenon is that the MWCNTs can prompt hydration reactions involving $\mathrm{C}_{3} \mathrm{~S}$ and $\mathrm{C}_{2} \mathrm{~S}$, transforming $\mathrm{C}_{3} \mathrm{~S}$ and $\mathrm{C}_{2} \mathrm{~S}$ into $\mathrm{C}-\mathrm{S}-\mathrm{H}$ gel. The XRD analysis results show that the differences in diffraction peak intensities are mainly affected by the MWCNT content in cement. Therefore, the presence of MWCNTs enhances the formation of $\mathrm{C}-\mathrm{S}-\mathrm{H}$ gel and facilitates the hydration process in cement samples.

\subsection{Pore structure analysis}

The pore structure determines the quality and performance of the MWCNT/cement nanocomposites. The mechanical properties are directly related to the pore structure, given that high porosity suggests a low compaction degree and inhibits the mechanical strength and durability of cement-based materials. The pore structures of the MWCNT reinforced cementitious materials are characterized using the total porosity and pore size distribution data. According to the literature, ${ }^{57}$ pores in cementitious materials are classified as innocuous pores (<20 $\mathrm{nm}$ in diameter), less harmful pores $(20-50 \mathrm{~nm})$, harmful pores (50-200 nm) and more harmful pores (>200 nm). Here, it's worth noting that decreasing the ratio of pores greater than $50 \mathrm{~nm}$ in diameter is more favorable for improving the performances of MWCNT/cement nanocomposites. ${ }^{58}$

The total porosity and pore size distribution data from MWCNT/cement composites of different ages are shown in Fig. 8. The total porosities of samples P, C1, C2, C3, and C4 after 1 day are $35.2 \%, 33.9 \%, 32.7 \%, 30.5 \%$ and $32.2 \%$, respectively, while the total porosities of samples P, C1, C2, C3, and C4 after 28 days are $19.4 \%, 17.7 \%, 16.3 \%, 17.0 \%$ and $18.6 \%$, respectively. The results in Fig. 8 show that the total porosity of every sample tends to reduce as the curing age increases; the addition of MWCNTs also leads to a decrease in the total porosity compared with a pure cement sample $\mathrm{P}$ of the same curing age. 

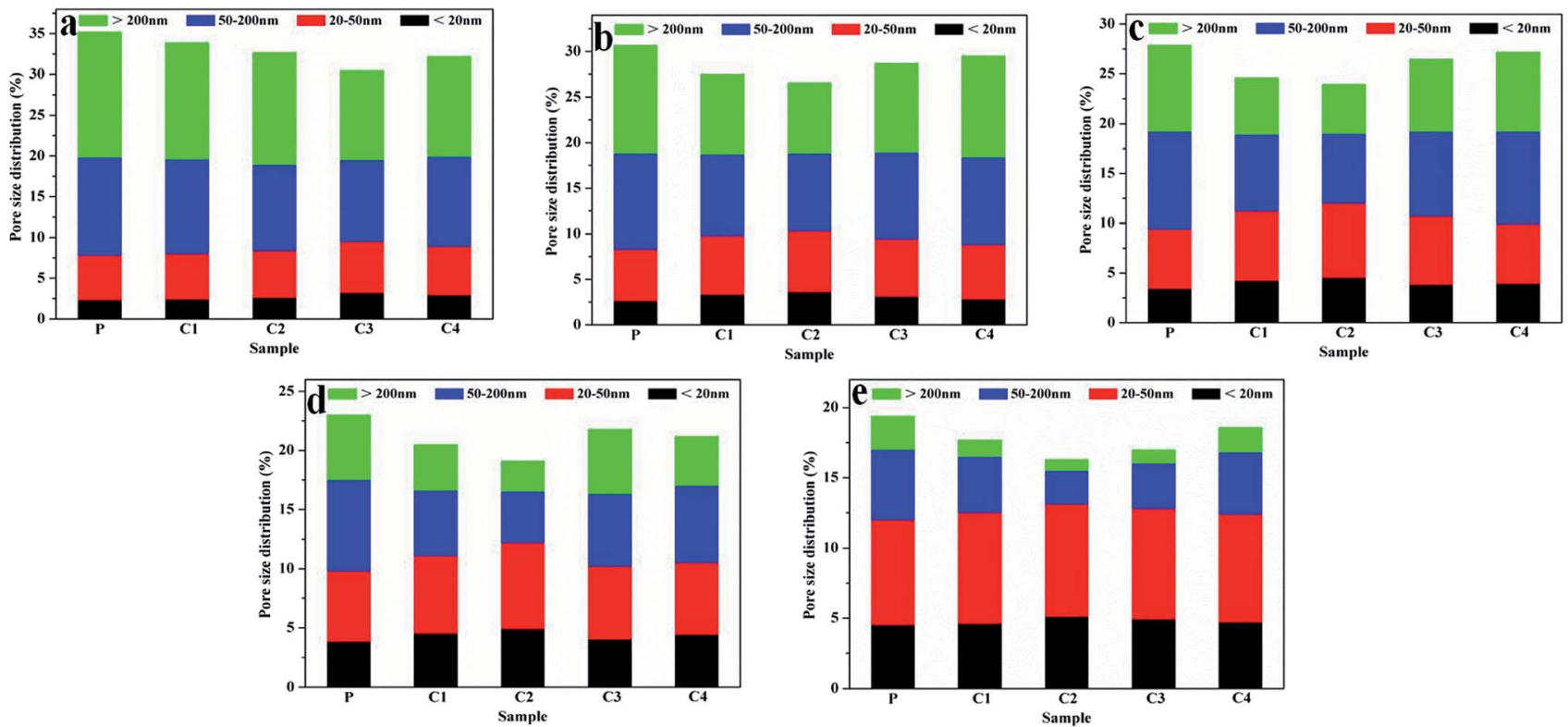

Fig. 8 The total porosity and pore size distribution data of MWCNT/cement samples at different ages ((a): 1 day; (b): 3 days; (c): 7 days; (d): 14 days; (e): 28 days).

The trends are similar to the changes in mechanical properties. The lowered total porosities indicate that the pores in the MWCNT/cement samples are filled with the continuously formed hydration products; MWCNTs can fill the large voids and promote the hydration process between voids and unhydrated cement, making the cement composites more compact.

The incorporation of MWCNTs not only affects the porosities of the cement composites, but it also influences the pore size distribution at different curing ages. As can be seen from Fig. 8a, the percentage of pores larger than $50 \mathrm{~nm}$ in diameter is greater than that of pores with a diameter less than $50 \mathrm{~nm}$. Compared with the pure cement sample P, the percentage of pores with a diameter larger than $50 \mathrm{~nm}$ decreases by $5.5 \%, 11.3 \%, 23.4 \%$ and $15.0 \%$ in samples $\mathrm{C} 1$, $\mathrm{C} 2$, C3, and $\mathrm{C} 4$, respectively, after 1 day. As the curing age increases from 3 to 14 days (Fig. $8 \mathrm{~b}-\mathrm{d}$ ), the percentage of pores with a diameter larger than $50 \mathrm{~nm}$ in the cement composites decreases gradually and the percentage of pores less than $50 \mathrm{~nm}$ in diameter increases accordingly. Meanwhile, the ratio of pores smaller than $50 \mathrm{~nm}$ in samples C1, $\mathrm{C} 2, \mathrm{C} 3$, and $\mathrm{C} 4$ is more than that in the pure cement sample P. After 28 days, the pores with a diameter of less than $50 \mathrm{~nm}$ in samples P, C1, C2, C3 and C4 account for $61.9 \%, 70.6 \%$, $80.4 \%, 75.3 \%$, and $66.7 \%$ of the total porosity, respectively (Fig. 8e). The results indicate that the number of pores with a diameter of less than $50 \mathrm{~nm}$ in the cement samples increases significantly compared to at an early curing age, and MWCNT addition can reduce the proportion of pores larger than $50 \mathrm{~nm}$ in diameter at every curing age. The pore size distribution phenomenon can be ascribed to filling effects of MWCNTs and hydration products, facilitating the transformation of detrimental pores (diameter $>50 \mathrm{~nm}$ ) to less harmful pores (diameter $<50 \mathrm{~nm}$ ). Therefore, the presence of MWCNTs is able to optimize the pore size distribution within the matrix, further enhancing the mechanical strengths of the MWCNT/cement nanocomposites.

\subsection{Strengthening and toughening mechanism}

SEM images of the fracture surface morphologies of a pure cement sample and the MWCNT/cement sample C2 after 28 days are shown in Fig. 9. Fig. 9a exhibits a micrograph of the pure cement matrix, revealing typical brittle fractures with poor resistance towards rupturing or cracking. The major elemental components of the pure cement matrix are $\mathrm{Ca}, \mathrm{Si}, \mathrm{O}, \mathrm{Al}$, and $\mathrm{K}$ (Fig. S4a†). Fig. 9b presents an SEM image of a cement sample containing MWCNTs without dispersion. The MWCNTs are dispersed poorly in the matrix. The MWCNTs are intertwined with each other and there is a large MWCNT agglomerate area. At the same time, the interface defects between the MWCNTs and the cement matrix ultimately lead to a decrease in the strength of the cement composite. Fig. $9 c$ shows a micrograph of a MWCNT/cement sample with the assistance of TNWDIS for dispersion, with EDS spectra results shown in Fig. S4b. $\dagger$ The element $\mathrm{C}$ is found to be present in Fig. $\mathrm{S} 4 \mathrm{~b}, \uparrow$ demonstrating that the MWCNTs have been integrated into the cement matrix. It can be seen from Fig. $9 c$ that the MWCNTs are well dispersed in the cement matrix, and the ends of the MWCNTs are raised on the surface, indicating that the MWCNTs can be effectively dispersed. The surfactant TNWDIS weakens the van der Waals forces between the MWCNTs and enhances their chemical compatibility with the cement matrix, thus resulting in efficient debundling and the satisfactory dispersion of the MWCNTs in cement. In addition, fibrous MWCNTs are embedded in the cement sample by means of network filling, crack bridging and 

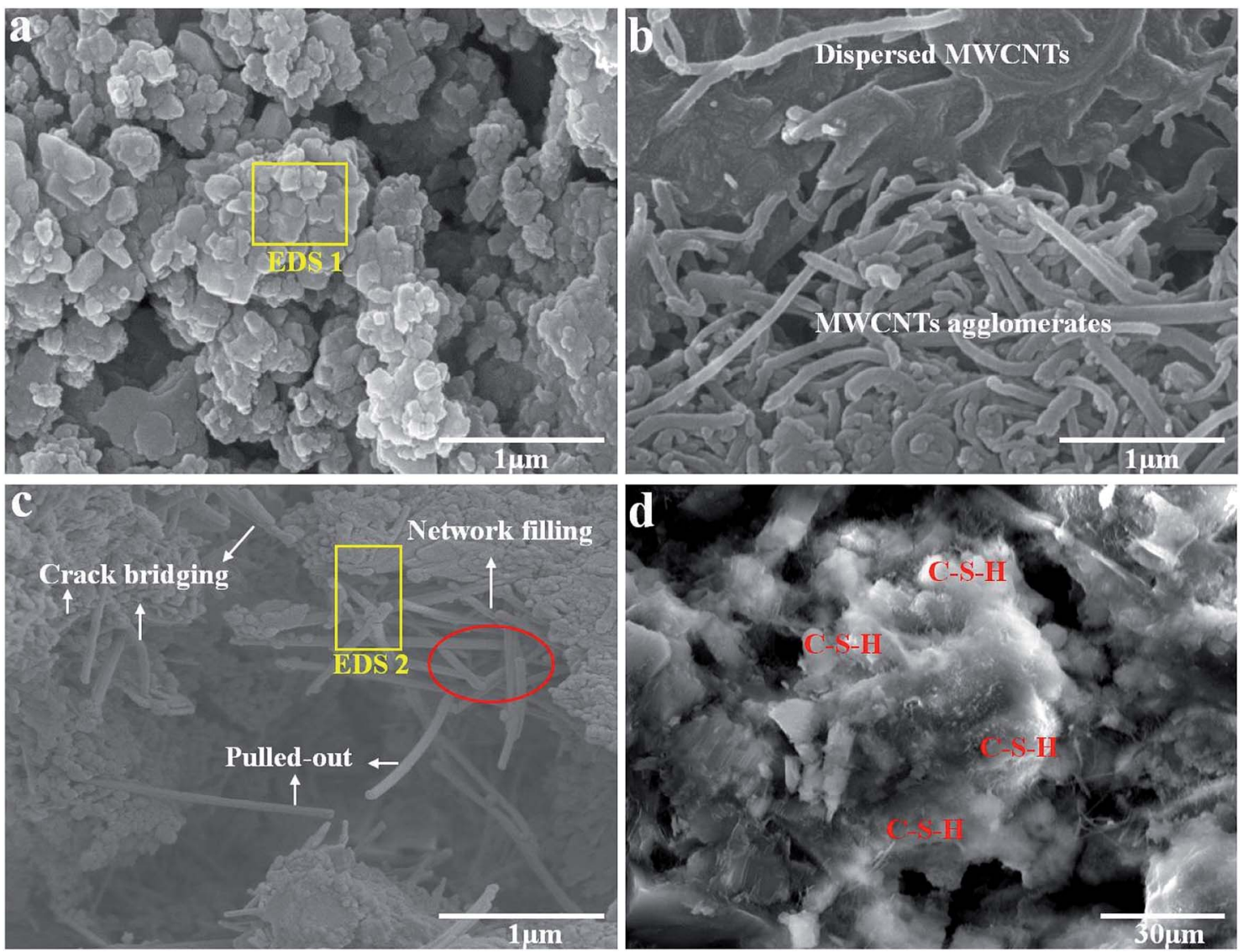

Fig. 9 SEM images of cementitious samples: (a) a pure cement matrix; (b) a cement sample containing MWCNTs without dispersion; (c) a cement sample containing MWCNTs dispersed with TNWDIS; and (d) the cement hydration products.

pulling out, to form a three-dimensional network structure. The network structure exhibits plastic deformation features, which can play a supporting role in the cement stability. When the MWCNT/cement sample is fractured by an external force, the direction of crack development is perpendicular to the MWCNTs; MWCNTs are pulled out and exfoliated from the cement matrix. During the pulling out process, the friction force between the MWCNTs and the cement matrix causes any energy for further crack development to be dissipated..$^{59}$ The presence of the MWCNTs inhibits cracks from propagating at the nanoscale, increases the connection area of the internal structure and provides high fracture resistance under an external load. ${ }^{60}$

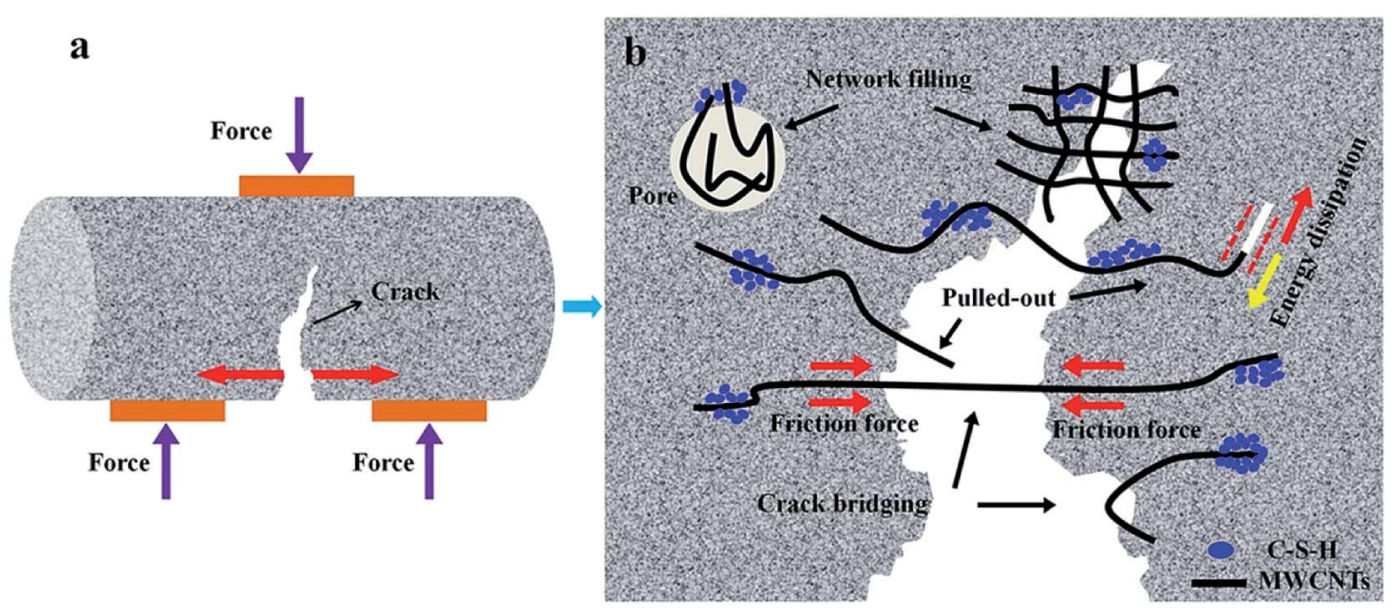

Fig. 10 The reinforcing and toughening mechanism of MWCNTs in cementitious nanocomposites under an external load: (a) a schematic illustration; and (b) a higher magnification schematic image. 
This is the mechanism behind the reinforcing and toughening properties of the MWCNTs (Fig. 10). Macroscopically, the mechanical properties of the cement nanocomposites are improved. Fig. 9d gives a microscopic view of the cement hydration products, and the hydration products are relatively abundant. It is noteworthy that the incorporation of MWCNTs facilitates the hydration of cement in the composite, which is in accordance with other literature studies. ${ }^{30,45}$ MWCNTs are assumed to serve as nucleation sites for the formation of $\mathrm{C}-\mathrm{S}-\mathrm{H}$ gel, and the $\mathrm{C}-\mathrm{S}-\mathrm{H}$ gel appears to form preferentially around the MWCNTs, so as to accelerate the formation rate of $\mathrm{C}-\mathrm{S}-\mathrm{H}$. Direct nucleation at the MWCNTs produces a dense $\mathrm{C}-\mathrm{S}-\mathrm{H}$ structure, which forms as a coating along the MWCNT surfaces (Fig. 10); this can enhance the load-transfer capacity between the MWCNTs and the cement matrix. Also, the MWCNTs serve as fillers in the voids, making the structures of the hydration products more compact.

\section{Conclusions}

In this paper, MWCNTs were applied to oil well cement to form MWCNT reinforced oil well cement-based nanocomposites for oil and gas well engineering. The effects of MWCNTs on the performances of cementitious nanocomposites were studied. TNWDIS is preferred as the dispersant for the MWCNTs. The fluidity of oil well cement slurry declines with an increase in the MWCNT content, and the addition of MWCNTs is controlled preferably below $0.50 \mathrm{wt} \%$ to meet the construction requirements for oil well cement slurry. For a cement sample with 0.05 wt $\%$ MWCNTs, the 28 day compressive strength is increased by $37.50 \%$ and the 28 day flexural strength is increased by $45.79 \%$ compared with pure cement. The elastic moduli of a $0.05 \mathrm{wt} \%$ MWCNT cementitious nanocomposite are reduced by $19.07 \%$ and $35.39 \%$, respectively, in uniaxial and triaxial mechanical experiments, showing an improvement in toughening effects compared with the pure cement matrix. XRD and pore structure analyses indicate that the addition of MWCNTs can accelerate the hydration process, increase the amount of hydration products and optimize the pore size distribution within the matrix. Meanwhile, MWCNTs can play a role in strengthening and toughening cement samples by means of network filling, pulling out, crack bridging and a $\mathrm{C}-\mathrm{S}-\mathrm{H}$ phase. The presence of MWCNTs provides a high resistance to fracture under an external load, prevents crack growth at the nanoscale and impedes the generation and propagation of micro-cracks. Therefore, the application of MWCNT reinforced nanomaterials in oil well cement will effectively facilitate the development of oil field cementing.

\section{Conflicts of interest}

There are no conflicts to declare.

\section{Acknowledgements}

The research described in this paper was financially supported by the National Natural Science Foundation of China (Grant No.
51674270), the National Science and Technology Major Project (2017ZX05009-003), the Major Project of the National Natural Science Foundation of China (No. 51490650) and the Foundation for Innovative Research Groups of the National Natural Science Foundation of China (Grant: No. 51521063).

\section{References}

1 I. P. G. Silva, A. A. Aguiar, V. P. Rezende, A. L. M. Monsores and E. F. Lucas, J. Pet. Sci. Eng., 2018, 161, 468-475.

2 C. Jia, Pet. Explor. Dev., 2017, 44, 1-10.

3 Q. Li, H. Xing, J. Liu and X. Liu, Petroleum, 2015, 1, 8-15.

4 G. Quercia, H. J. H. Brouwers, A. Garnier and K. Luke, Mater. Des., 2016, 96, 162-170.

5 M. Li, S. Deng, Y. Yu, J. Jin, Y. Yang and X. Guo, Constr. Build. Mater., 2017, 135, 59-67.

6 A. Srivastava, R. Ahmed and S. Shah, J. Pet. Sci. Eng., 2018, 170, 218-230.

7 J. Konkol and G. Prokopski, Constr. Build. Mater., 2016, 123, 638-648.

8 M. Heikal, H. El-Didamony, T. M. Sokkary and I. A. Ahmed, Constr. Build. Mater., 2013, 38, 1180-1190.

9 O. Sengul, Constr. Build. Mater., 2016, 122, 649-658.

10 S. S. Joshi, N. Thammishetti, S. S. Prakash and S. Jain, ACI Struct. J., 2018, 115, 1575-1588.

11 K. Holschemacher, T. Mueller and Y. Ribakov, Mater. Des., 2010, 31, 2604-2615.

12 S. A. Yildizel, O. Timur and A. U. Ozturk, Mech. Compos. Mater., 2018, 54, 251-256.

13 S. Naqvi, K. Mahmoud and E. El-Salakawy, Eng. Struct., 2017, 134, 376-389.

14 F. Pacheco-Torgal and S. Jalali, Constr. Build. Mater., 2011, 25, 575-581.

15 S. Fic, P. Brzyski and M. Szeląg, Ecol. Chem. Eng. S, 2013, 20, 899-907.

16 T. Nishiwaki, M. Koda, M. Yamada, H. Mihashi and T. Kikuta, J. Adv. Concr. Technol., 2012, 10, 195-206.

17 Y. Wang, H. C. Wu and V. C. Li, J. Mater. Civ. Eng., 2000, 12, 314-319.

18 S. Sun, X. Yu, B. Han and J. Ou, Constr. Build. Mater., 2013, 49, 835-840.

19 K. M. Liew, M. F. Kai and L. W. Zhang, Composites, Part A, 2016, 91, 301-323.

20 M. S. Konsta-Gdoutos, Z. S. Metaxa and S. P. Shah, Cem. Concr. Compos., 2010, 32, 110-115.

21 S. Sharma and N. C. Kothiyal, RSC Adv., 2015, 5, 52642.

22 L. Wang, D. Zheng, S. Zhang, H. Cui and D. Li, Nanomaterials, 2016, 6, 241.

23 L. Senff, D. Hotza, S. Lucas, V. M. Ferreira and J. A. Labrincha, Mater. Sci. Eng., A, 2012, 532, 354-361.

24 S. Song, L. Jiang, S. Jiang, X. Yan and N. Xu, Constr. Build. Mater., 2018, 164, 663-671.

25 Z. Yang, Y. Gao, S. Mu, H. Chang, W. Sun and J. Jiang, Constr. Build. Mater., 2019, 195, 415-422.

26 M. Oltulu and R. Şahin, Mater. Sci. Eng., A, 2011, 528, 70127019. 
27 S. Kawashima, P. Hou, D. J. Corr and S. P. Shah, Cem. Concr. Compos., 2013, 36, 8-15.

28 J. M. Makar and J. J. Beaudoin, carbon nanotubes and their applications in the construction industry. Proceedings of the 1st International Symposium on Nanotechnology in Construction, Paisley, Scotland, UK, 23-25 June, 2003, ed. Bartos P.J.M., Hughes J.J., Trtik P. and Zhu W., Royal Society of Chemistry, London, UK, 2004, pp. 331-341.

29 M. F. Yu, O. Lourie, M. J. Dyer, K. Moloni, T. F. Kelly and R. S. Ruoff, Science, 2000, 287, 637-640.

30 W. Li, W. Ji, F. Torabian Isfabian, Y. Wang, G. Li, Y. Liu and F. Xing, Nanomaterials, 2017, 7, 185.

31 L. J. Sudak, J. Appl. Phys., 2003, 94, 7281-7287.

32 A. L. Materazzi, F. Ubertini and A. D'Alessandro, Cem. Concr. Compos., 2013, 37, 2-11.

33 B. Han, S. Sun, S. Ding, L. Zhang, X. Yu and J. Ou, Composites, Part A, 2015, 70, 69-81.

34 S. Xu, J. Liu and Q. Li, Constr. Build. Mater., 2015, 76, 16-23.

35 M. S. Konsta-Gdoutos and C. A. Aza, Cem. Concr. Compos., 2014, 53, 162-169.

36 K. M. Liew, M. F. Kai and L. W. Zhang, Compos. Struct., 2017, 160, 81-88.

37 M. Szelag, Nanomaterials, 2017, 7, 267.

38 M. d. C. Camacho, O. Galao, F. J. Baeza, E. Zornoza and P. Garcés, Materials, 2014, 7, 1640-1651.

39 F. Sanchez and K. Sobolev, Constr. Build. Mater., 2010, 24, 2060-2071.

40 S. Kumar, P. Kolay, S. Malla and S. Mishra, J. Mater. Civ. Eng., 2012, 24, 84-91.

$41 \mathrm{~T}$. Kowald, Influence of surface-modified carbon nanotubes on ultra-high performance concrete. Proceedings of the international symposium on ultra high performance concrete. University of Kassel, Germany, 13-15 September, 2004, ed. Schmidt M.; Fehling E. and Geisenhanslüke C., Kassel University Press, Kassel, Germany, 2004, pp. 195-202.

42 T. Nochaiya and A. Chaipanich, Appl. Surf. Sci., 2011, 257, 1941-1945.

43 Y. Sáez de Ibarra, J. J. Gaitero, E. Erkizia and I. Campillo, Phys. Status Solidi A, 2006, 203, 1076-1081.
44 X. L. Xie, Y. W. Mai and X. P. Zhou, Mater. Sci. Eng. R Rep., 2005, 49, 89-112.

45 F. T. Isfahani, W. Li and E. Redaelli, Cem. Concr. Compos., 2016, 74, 154-163.

46 W. W. Li, W. M. Ji, Y. C. Wang, Y. Liu, R. X. Shen and F. Xing, Materials, 2015, 8, 8780-8792.

47 F. Ubertini, A. L. Materazzi, A. D'Alessandro and S. Laflamme, Eng. Struct., 2014, 60, 265-275.

48 S. Musso, J. M. Tulliani, G. Ferro and A. Tagliaferro, Compos. Sci. Technol., 2009, 69, 1985-1990.

49 G. Y. Li, P. M. Wang and X. Zhao, Carbon, 2005, 43, 12391245.

50 A. Cwirzen, K. Habermehl-Chirzen and V. Penttala, Adv. Cem. Res., 2008, 20, 65-73.

51 ASTM C305-14, Standard practice for mechanical mixing of hydraulic cement pastes and mortars of plastic consistency, ASTM International, West Conshohocken, PA, USA, 2014.

52 ASTM C349-14, Standard test method for compressive strength of hydraulic-cement mortars (using portions of prisms broken in flexure), ASTM International, West Conshohocken, PA, USA, 2014.

53 ASTM C348-14, Standard test method for flexural strength of hydraulic-cement mortars, ASTM International, West Conshohocken, PA, USA, 2014.

54 F. Zhou, Y. Wang, W. Wu, T. Jing, S. Mei and Y. Zhou, Sci. Rep., 2016, 6, 38000.

55 J. M. Makar and G. W. Chan, J. Am. Ceram. Soc., 2009, 92, 1303-1310.

56 Y. J. Yim and S. J. Park, Composites Research, 2015, 28, 361365.

57 W. J. Long, H. D. Li, C. L. Fang and F. Xing, Nanomaterials, 2018, 8, 31.

58 H. Ma, B. Xu, J. Liu, H. Pei and Z. Li, Mater. Des., 2014, 64, 497-502.

59 P. Yu, Z. Wang, S. Lu and P. Lai, J. Nanosci. Nanotechnol., 2019, 19, 163-169.

60 Z. Qu and G. Wang, J. Appl. Polym. Sci., 2012, 124, 403-411. 